\title{
Exploring the Regulatory Mechanism of Stress Responses in the Paraventricular Nucleus of the Hypothalamus: Backgrounds and Future Perspectives of Corticotropin-Releasing Factor-Modified Yellow Fluorescent Protein-Knock-In Mouse
}

\author{
Keiichi ITOI* \\ Graduate School of Information Sciences and Graduate School of Medicine, \\ Tohoku University, Sendai 980-8579, Japan
}

Received July 28, 2015; final version accepted July 31, 2015

\begin{abstract}
Corticotropin-releasing factor (CRF) plays a central role in the stress response by regulating the hypothalamicpituitary-adrenal axis. In order to unravel unsolved issues underlying the regulatory mechanisms for CRF neurons, modified yellow fluorescent protein (Venus) gene was inserted into the CRF gene in frame, and CRF neurons were visualized by the Venus fluorescence. Venus expression is overlapped with CRF expression in most brain regions, including the paraventricular nucleus of the hypothalamus (PVH). This mouse is a useful tool especially for conducting electrophysiological recordings from CRF neurons. In the first half of the present review, the backgrounds of the generation of the mouse are described based on the previous literature: first, the anatomical distribution of CRF-immunoreactive neurons in the rat brain is overviewed, and then the knowledge on the electrophysiological properties of the parvocellular neuroendocrine neurons that constitute a subpopulation of neurons in the PVH (including PVH-CRF neurons) is described. These sections may help the readers in understanding the purpose of generating the CRF-Venus mouse. In the second half of the manuscript, the distribution of Venus-expressing neurons is characterized in the CRF-Venus mouse, and preliminary results of electrophysiological recordings from the Venus-expressing neurons are shown. CRF driver mouse lines are also referred to as a means for the CRF neuron-selective gene transfer or targeting. Novel mouse lines may serve as tools for disclosing the regulatory mechanisms for CRF neurons in the $\mathrm{PVH}$, as well as other brain regions.
\end{abstract}

KEYWORDS: mouse, fluorescent proteins, patch-clamp, immunofluorescence, noradrenaline

\section{Introduction}

The corticotropin-releasing factor (CRF)-containing neurons located in the paraventricular nucleus of the hypothalamus (PVH) play a pivotal role in the regulation of the hypothalamic-pituitary-adrenal (HPA) axis (Fig. 1). CRF neurons are regulated by a variety of neural inputs and humoral factors [1]. For example, systemic stress signals are conveyed primarily to the medulla oblongata, via visceral afferents, and then they are transmitted to the PVH-CRF neurons; the ascending noradrenergic (NAergic) system is implicated in this pathway. Glutamate-containing (glutamatergic) and $\gamma$-aminobutyric acid (GABA)-containing (GABAergic) neurons make direct synaptic contacts with CRF neurons and may take part in determining the excitability of CRF neurons [2]. Interestingly, NAergic inputs may modulate the transmitter discharge from the local glutamatergic or GABAergic neurons [2]. Part of the glutamatergic and GABAergic afferents derive from the regions outside the hypothalamus, where the stress signals, transmitted mainly from the limbic structures, are relayed to the hypothalamus [3]. Glucocorticoid (GC) is secreted from the adrenals in response to stress exposure; besides its essential role in protecting an organism from stressors, GC also regulates CRF neurons in the PVH by a negative feedback mechanism [1-3]. PVH is also a center of the sympathetic and parasympathetic autonomic nervous systems [3], and the interplay between the neuroendocrine and autonomic systems has been proposed at various levels of the brain including the PVH itself [4]. A brief summary of topographical relationship between the two systems are presented schematically in Fig. 1.

Recently, CRF-modified yellow fluorescent protein (Venus) knock-in mouse was generated in my laboratory with the collaboration of Dr. Kenji Sakimura at Niigata University, for the purpose of overcoming previous difficulties in carrying out cellular physiological studies [5]. The knowledge of the previous research works may help the readers in understanding the reason why fluorescent labeling of CRF neurons is important for future research; the background to the generation of CRF-Venus mouse is described in the first half of the present review. In the second half, characteristics of the CRF-Venus mouse will be presented, and this may lead to future perspectives of the mouse as a tool for studying CRF neurons. 


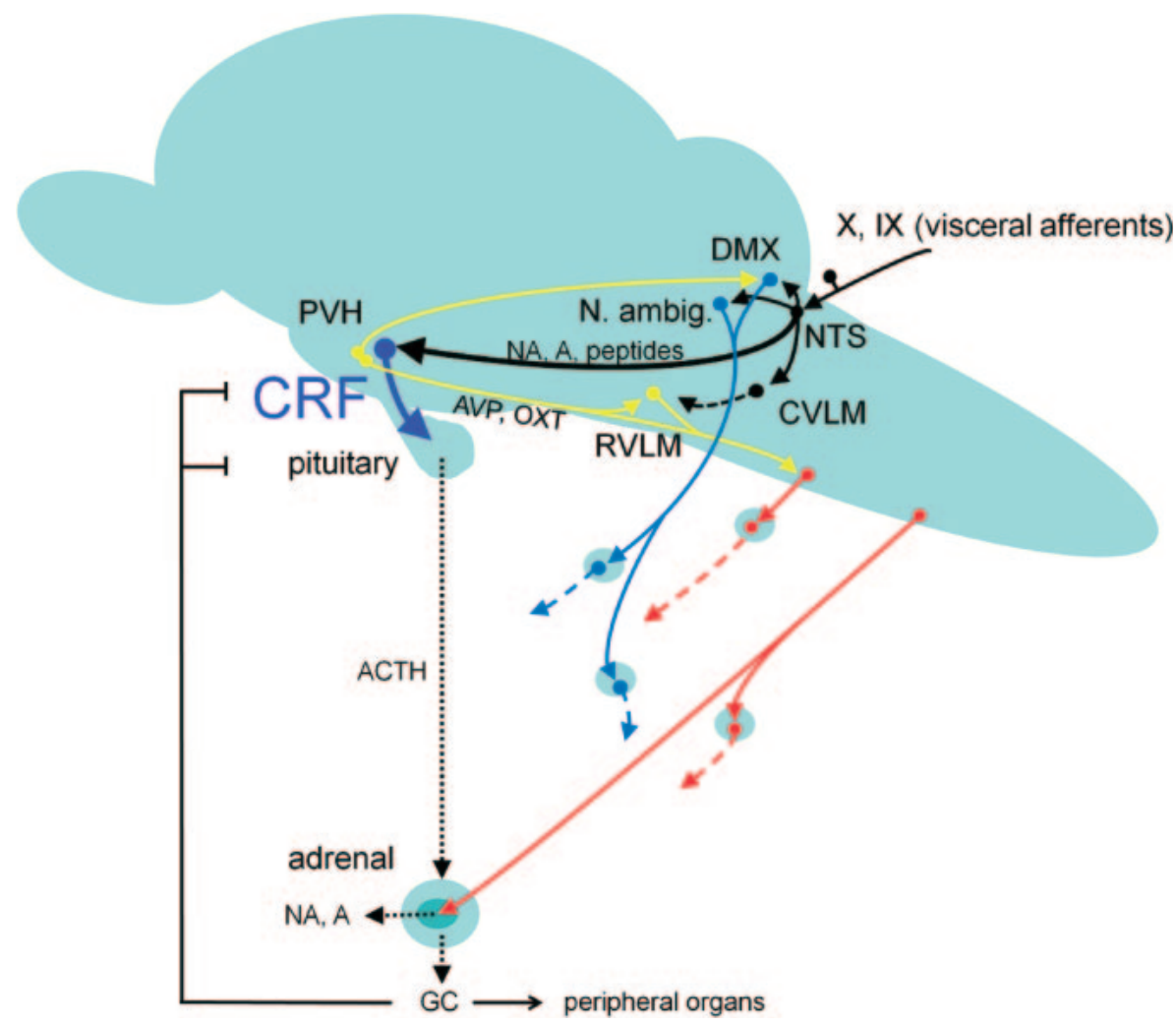

Fig. 1. CRF neurons as a center of the hypothalamic-pituitary-adrenal axis and their interplay with the autonomic nervous system

The CRF neurons in the paraventricular nucleus of the hypothalamus (PVH) play a central role in the regulation of the hypothalamic-pituitary-adrenal axis. CRF neurons receive inputs from many brain regions, and most extensively studied, among other neurons, are the ascending catecholaminergic neurons originating from the nucleus of the solitary tract (NTS). These neurons contain noradrenaline (NA) or adrenaline (A), as well as various neuropeptides. The NTS receives visceral afferents via the vagal (X) and glossopharyngeal (IX) nerves that convey signals from the visceral organs and great vessels. Thus, the visceral signals are transmitted to the PVH and regulate the output levels of CRF neurons, besides regulating outputs from the medullary autonomic center such as the rostral ventrolateral medulla (RVLM). Subpopulations of the vasopressin (AVP) and oxytocin (OXT) neurons in the PVH are pre-autonomic neurons that project to the RVLM and sympathetic preganglionic neurons in the spinal cord. Part of the pre-autonomic neurons in the PVH also projects to the dorsal motor nucleus of vagus (DMX). GC, glucocorticoid; ACTH, adrenocorticotropin; N. ambig., nucleus ambiguous; CVLM, caudal ventrolateral medulla; yellow lines, pre-autonomic neurons; light blue lines, parasympathetic neurons; red lines, sympathetic neurons (solid lines are preganglionic and dotted lines postganglionic). The black dotted line represents $\gamma$-aminobutyric acid-containing interneurons from the CVLM to RVLM.

Thorough examination of Venus expression in the whole brain is an essential part of the characterization of the CRFVenus mouse because tissue-selective expression of a gene may not always be attained even via the knock-in strategy, and it is indispensable to check for the possible 'off-target' (ectopic) expression of the Venus gene. The following section, 'Anatomical distribution of CRF neurons in the brain', will provide readers with resources for better understanding the topography of Venus-expressing neurons in later sections. 'Regulatory mechanisms for CRF neurons in the PVH' is discussed in the next section from an electrophysiological viewpoint since the fluorescent visualization is of greatest benefit to the electrical recordings from CRF neurons. As an 'Application of novel technologies for exploring CRF neurons', CRF-Venus mouse is characterized and compared to other mouse lines for appropriate uses in future experiments. CRF driver mouse lines are also referred to as a means for the CRF neuron-selective gene transfer or targeting. The final section presents a summary of the present review.

\section{Anatomical distribution of CRF neurons in the brain}

\subsection{Overall anatomy of CRF-producing neurons in the brain}

Earlier studies in the 1980s revealed major sites which contain CRF-immunoreactive (ir) cell bodies and axons throughout the rat brain [6-8]: brain regions where conspicuous CRF-ir cell bodies were observed include medial and median preoptic areas, bed nucleus of the stria terminalis (BNST), PVH, lateral hypothalamus, cingulate cortex, sensorimotor cortices (mainly layers II and III), piriform cortex, central nucleus of amygdala (CeA), laterodorsal tegmental nucleus (LDTg), pedunclopontine tegmental nucleus (PPTg), periaqueductal gray (PAG), Barrington's nucleus, lateral parabrachial nucleus (LPB), nucleus of the solitary tract (NTS), and ventrolateral reticular formation. 
CRF-containing fibers are present in broader brain regions including the areas containing the CRF cell bodies.

The CRF-ir axons are observed throughout the forebrain, midbrain, and hindbrain structures, and sometimes the fibers appear to traverse a long distance between different brain regions [6-8]. However, it is difficult to know from which region these axons originate and to which direction they are running, so the physiological role(s) of most CRFcontaining projection neurons is still elusive. Therefore, the 'connectome' of the CRF neuronal system in the brain has yet to be disclosed.

In several groups of CRF-containing neurons, however, the axon terminals can be traced back to the cell bodies of their origin; for example, terminals in the external layer of the median eminence (ME) derive from the PVH-CRF neurons $[6,7,9]$. These fibers terminate on the capillary walls in the external layer of the ME, where CRF peptide is secreted [6]. Then, CRF is carried to the anterior pituitary via the pituitary portal veins and stimulates the synthesis and secretion of adrenocorticotropin (ACTH) [10]. Prominent expression of CRF is observed in Barrington's nucleus which is the pontine micturition center and projects directly to parasympathetic preganglionic neurons in the lumbosacral spinal cord [11]. CRF is also contained in the inferior olivary nucleus (IO), which is the source of the ascending fibers to the cerebellum that modulate the long-term depression [12].

Our knowledge on the distribution of CRF-ir neurons in the human brain is limited [13-17]. The cytoarchitectonics of the human PVH is different from that of rodents, and parvocellular and magnocellular neurons are interspersed within the same area [14]. CRF-immnoreactivity was present only in the parvocellular neurons and did not appear to be present in either the magnocellular neurons in the PVH or supraoptic nucleus in the human hypothalamus [13,14]. CRF-ir axons could also be traced to the ME in humans, where they form a dense network of terminals around the portal capillaries [16]. CRF-ir cell bodies were also observed in the periventricular nucleus and infundibular nucleus of human hypothalamus, the latter being the equivalent of rodent arcuate nucleus [17].

The precise map of the CRF-ir neuronal system has not yet been reported in the mouse because CRF neurons could not be stained satisfactorily with any anti-CRF antibody available. In the mouse, cytoplasmic CRF concentration could possibly be smaller, compared to that in rats, since CRF neurons can be stained in the mouse PVH following an injection of colchicine intracerebroventricularly to block the axonal transport and increase CRF content in the perikarya [5, 18] (vide infra). However, CRF neurons, outside the PVH, are difficult to stain even after colchicine injection (possibly, colchicine could not readily diffuse into areas far from the ventricular wall). Recently, Alon and colleagues generated a transgenic mousse line, by the bacterial artificial chromosome (BAC) strategy, in which the green fluorescent protein (GFP) is expressed under the promoter of CRF [19]; they mapped the distribution of GFP-ir (putative CRF-containing) cell bodies in the mouse brain [19]. The distribution of CRF mRNA-expressing neurons in the mouse brain was reported in a few previous reports [19,20] and Allen's Brain Atlas (http:// mouse.brain-map.org/).

\subsection{CRF in the PVH as a neuroendocrine peptide}

The CRF neurons in the PVH are studied most extensively because they play a central role in the regulation of the HPA stress axis [10]. The PVH comprises three types of neurons: the magnocellular neuroendocrine neurons, parvocellular neuroendocrine neurons, and pre-autonomic (parvocellular) neurons [21]. In the rat, magnocellular neuroendocrine neurons are localized in a more dorsolateral portion of the PVH (mainly in the posterior magnocellular subdivision), while the parvocellular neuroendocrine neurons are located in the medial region of the PVH (mainly the medial parvocellular subdivision). A less prominent parvocellular cell group is present in the periventricular nucleus which is medial to the medial parvocellular subdivision and adjacent to the wall of the 3rd ventricle [6].

In the rat PVH, the magnocellular neurons are greater in size compared to the parvocellular neurons, but it is not always easy to discriminate the former from the latter morphologically. The magnocellular neurons comprise vasopressin (AVP)- and oxytocin (OXT)-containing neurons that project to the posterior pituitary where the neuropeptides are secreted into the systemic circulation [21]. The parvocellular neuroendocrine neurons in the PVH are smaller in size and comprise multiple populations of neuroendocrine neurons [CRF-, thyrotropin-releasing hormone (TRH)-, and somatostatin-synthesizing neurons] that project to the ME [21]. To help understand the topography of CRF neurons in the PVH, distribution of CRF mRNA in the rat PVH, together with that of AVP mRNA, is shown in Fig. 2; the region where CRF mRNA is expressed corresponds to the parvocellular subdivision, and the region with AVP mRNA to the magnocellular subdivision. Although not shown in Fig. 2, TRH and somatostatin are also expressed in the parvocellular subdivision, and OXT in the magnocellular subdivision as described above.

The pre-autonomic neurons are distributed in the dorsomedial 'cap', and ventral-most, as well as the extreme posterior, region of the rat PVH [2,22]; these neurons are not neurosecretory and project to brain stem [the rostral ventrolateral medulla (RVLM) and dorsal motor nucleus of the vagus (DMX)] and the lateral column of the spinal cord which are involved in sympathetic or parasympathetic control [23-25] (Fig. 1). In the mouse, the margins between subpopulation of the neuronal species in the PVH are more ambiguous [26]. In addition, the differences in sizes of cell bodies are not as great between the 'parvo-' and 'magnocellular' neurons as they are in the rat PVH, making it more difficult to discriminate the 'parvo-' from the 'magnocellular' neurons.

CRF neurons in the PVH have a potency to express AVP [27-29], and co-expression of AVP in the parvocellular CRF neurons becomes most conspicuous in a chronically GC-deficient state [27-29]. After bilateral adrenalectomy, a 


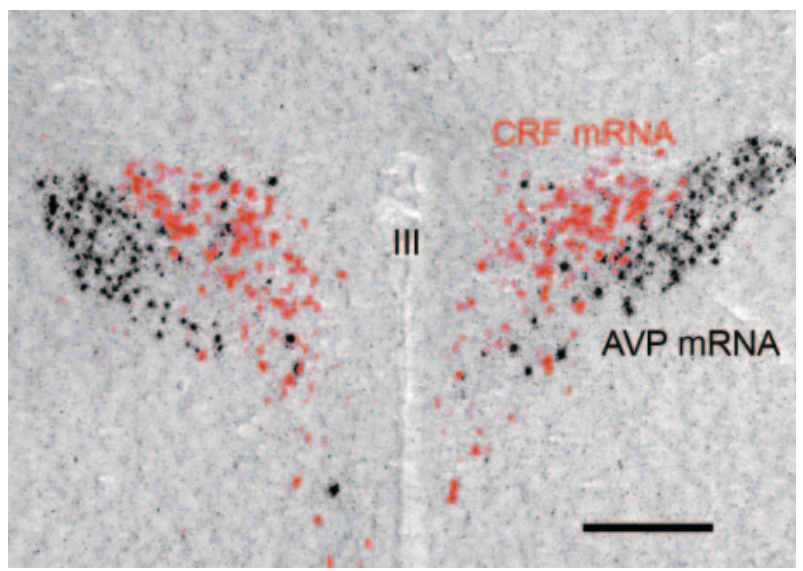

Fig. 2. Distribution of CRF mRNA and AVP mRNA in the rat PVH

Distributions of CRF mRNA and AVP mRNA are identified by in situ hybridization histochemistry using radiolabeled probes. CRF mRNA is in the parvocellular subdivision, while AVP mRNA in the magnocellular subdivision. In a basal (unstressed) state, very few CRF neurons co-express AVP, but after bilateral adrenalectomy, a considerable proportion of CRF neurons start expressing AVP (not shown). III, third ventricle. Scale bar $=250 \mu \mathrm{m}$.

significant proportion of CRF-ir neurons began expressing AVP-immunoreactivity in the rat [27-29] and mouse [5]. Co-localization of AVP in the PVH-CRF neurons was also observed in post-mortem human brain [14,17]. In the rat, $\mathrm{CRF}$ and AVP are co-stored in the same secretory granules in nerve terminals at the ME [30] and exert a synergistic effect on ACTH secretion [31,32]. Therefore, co-secretion of AVP together with CRF may help facilitate the release of ACTH in the anterior pituitary in GC deficient conditions.

\section{Regulatory mechanisms for CRF neurons in the PVH}

CRF neurons in the PVH are regulated by neural inputs and humoral factors $[1,3]$. The local neural circuits within the PVH contain the glutamatergic and GABAergic neurons [2]. The glutamatergic or GABAergic inputs were identified in both magno- [33,34] and parvocellular neurons in the PVH [35]. Albeit the presence of glutamatergic projection neurons from outside the $\mathrm{PVH}$ [3], a considerable proportion of glutamatergic cell bodies are presumed to be present in the PVH [33]. GABAergic cell bodies, however, cannot be identified within the PVH; the GABAergic neurons are present in the peri-PVH regions [35], which circumscribe the $\mathrm{PVH}$, as well as outside the $\mathrm{PVH}$, including the lateral hypothalamic area, BNST, anterior hypothalamic area, medial preoptic area, and dorsomedial hypothalamus $[3,36]$.

There are numerous populations of projection neurons to the PVH-CRF neurons from multiple brain regions $[1,3,37]$. Among these projection neurons, the ascending NAergic neurons, originating from the brain stem, have been studied most extensively $[1,3,37,38]$. In the rat, the parvocellular subdivision of the $\mathrm{PVH}$, where CRF neurons are present, receives NAergic afferents mostly from the A2 region in the NTS [39]. It was proposed that ascending catecholaminergic pathways may mediate systemic stress responses [3]. Several neuropeptides are colocalized with noradrenaline (NA) in the brain stem, and these peptides may modulate the action of NA at the PVH [40]. The NAergic projection from the locus ceruleus (A6) is also postulated to convey signals to the PVH indirectly via the dorsal medial prefrontal cortex and stimulate CRF neurons by disinhibition of the GABAergic afferents to the PVH-CRF neurons [41].

The GCs play a key role in the regulation of CRF neurons under acute or chronic stress via a negative feedback mechanism $[1,3,37]$. GCs are the ligands for the GC receptors (GRs), and the ligand-bound GRs exert their effects on CRF gene expression (the genomic effects of GCs). GCs also exert non-genomic effects on CRF neurons [35, 42], and endocannabinoid signaling may mediate part of the GC-mediated negative feedback regulation of the HPA-axis $[35,42,43]$. In the following sections, the regulatory mechanisms for the CRF neurons will be discussed from the electrophysiological viewpoints. Molecular mechanisms of CRF gene expression are discussed in an excellent review by Aguilera in the present issue of IIS [44].

\subsection{Differentiation of subpopulation of neurons in the PVH}

In the rat $\mathrm{PVH}$, the distribution of parvocellular neurons differs from that of magnocellular neurons, and the somata of parvocellular neurons are smaller than those of magnocellular neurons (vide supra). However, it is difficult to differentiate the former from the latter only by morphological characteristics. Tasker and colleagues developed a method to differentiate subpopulations of neurons in the PVH electrophysiologically [45-47]. The magnocellular neurons are grouped into the type I, and parvocellular neurons type II and type III; type II neurons correspond to the 
A

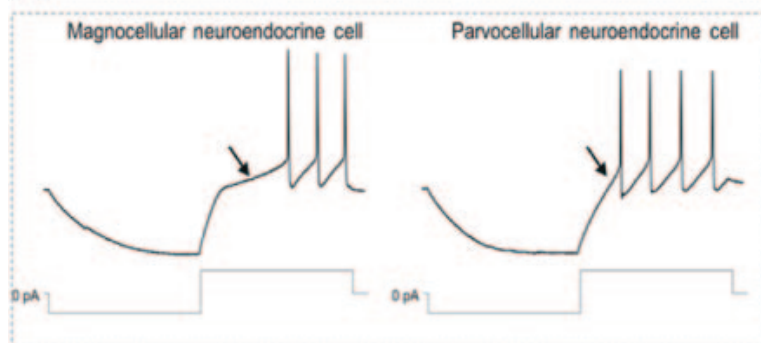

After Luther \& Tasker, 2000
B

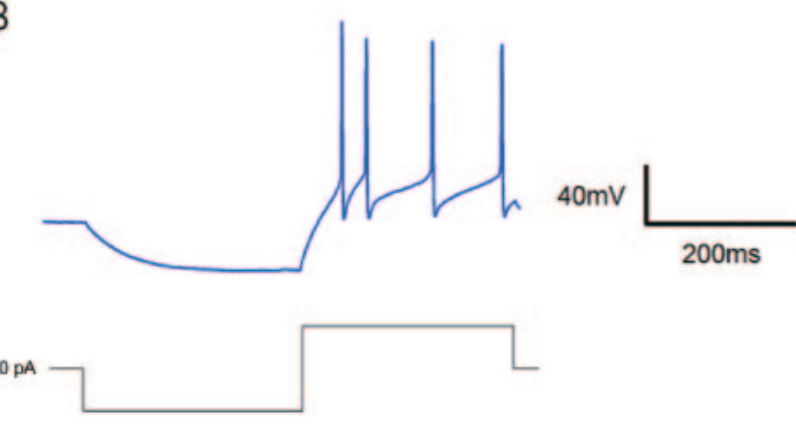

Fig. 3. Electrophysiological differentiation between the magnocellular neuroendocrine neurons and parvocellular neuroendocrine neurons in the PVH

A. Tasker and colleagues have developed a method for discriminating magnocellular neuroendocrine neurons from parvocellular neuroendocrine and pre-autonomic neurons in the rat PVH. The whole-cell clamp was carried out using an acute hypothalamic slice, and the time course of the initial phase of firing, elicited by a depolarization current, was observed under a current-clamp mode. In the magnocellular neurons, the transient outward rectification (arrow in the left recording) was observed by applying a depolarization current immediately after hyperpolarization. On the other hand, no transient outward rectification was observed in parvocellular neurons (arrow in the right recording). Reproduced from Levy and Tasker, 2012 [2], under the permission from Frontiers in Cellular Neuroscience. B. In the PVH of the CRF-Venus knock-in mouse, Venus-expressing neurons showed no transient outward rectification, confirming that they are parvocellular neurons [5] (for detail, see text).

parvocellular neuroendocrine neurons, and type III neurons to the pre-autonomic neurons. This classification was initially made in the rat PVH, but an identical method can be applied to the mouse PVH (Fig. 3). Both magno- and parvocellular neurons have resting membrane potential of approximately $-60 \mathrm{mV}$. The intracellular potential was monitored under a current clamp mode, and then, the cell was hyperpolarized from the basal state to $-100 \mathrm{mV}$ (the conditioning step), after which it was depolarized in a step-wise manner; the cells showing the transient outward rectification was designated as type I, and those not showing it as type II or type III [2,46, 47]. Luther and Tasker [46] demonstrated that the outward rectification that delays action potential generation [45] was attributed to the predominant expression of the voltage-gated potassium currents (an A-type $\mathrm{K}^{+}$current: $\mathrm{I}_{\mathrm{A}}$ ) in type I neurons. Type III neurons generated a low-threshold $\mathrm{Ca}^{2+}$ spike and T-type $\mathrm{Ca}^{2+}$ current $\left(\mathrm{I}_{\mathrm{T}}\right)[2,47,48]$. Although the electrophysiological differentiation is not an absolute means, the method contributed greatly in characterizing the parvo- and magnocellular neurons in the PVH.

\subsection{Glutamatergic, GABAergic, and NAergic inputs}

In the rat and mouse PVH, either glutamatergic excitatory post-synaptic currents (EPSCs) [35] or GABAergic inhibitory post-synaptic currents (IPSCs) [49] can be recorded in patch-clamped parvocellular neurons, under a voltageclamp mode. The glutamatergic and GABAergic neurons comprise the local hypothalamic circuits to regulate the neuroendocrine CRF neurons. Among the projection neurons that innervate the PVH-CRF neurons, NAergic neurons have been studied most extensively $[1,37,38]$ as was described above. NAergic axon terminals run in close apposition to CRF neurons [50] and make direct synaptic contacts with CRF neurons [51], as demonstrated by electron microscopic immunocytochemical method using an antibody against tyrosine hydroxylase. NA has been shown to stimulate the PVH-CRF neurons in vivo, in which ablation of NA inputs to the PVH inhibited stress-induced activation of CRF neurons [52]. NA increased CRF gene expression and secretion of ACTH in a dose-dependent manner when injected directly to the PVH of conscious rats [53,54].

The effect of NA on the electrophysiological properties of parvocellular neurons was examined by application of NA in the bath solution. The effects of NA were bimodal, excitatory or inhibitory, depending on the neuron being recorded [55]. NAergic excitation was blocked by prazosin, an $\alpha_{1}$-adrenoceptor antagonist, whereas NAergic inhibition was blocked by yohimbine, an $\alpha_{2}$-adrenoceptor antagonist. In adrenalectomized rats, the proportion of neurons excited by NA increased, and supplementation with corticosterone reversed the potentiation of NAergic excitation [55]. Thus, NA may act in either an excitatory or inhibitory manner on the parvocellular neurons, and GC increases the excitability of these neurons in response to NA. Daftary and colleagues [56] showed that bath application of NA increased the frequency of excitatory post-synaptic potentials (EPSPs) in a subpopulation of parvocellular neurons, and the EPSP was blocked by ionotropic glutamate receptor antagonists. The NA-induced increase in EPSC frequency was mediated by $\alpha_{1}$-adrenoceptors since it was abolished by prazosin. In addition, the NA-induced glutamate release was spikedependent [56]. In another subpopulation of parvocellular neurons, NA induced hyperpolarization that was blocked by the $\beta$-adrenoceptor antagonist, propranolol. They argue that this apparently inhibitory effect may be caused by a direct postsynaptic action of NA [56].

Since the stimulatory action of NA on CRF gene expression was shown in earlier studies [53,54], and NAergic neurons may make direct synaptic contact with the CRF neurons [50,51], NA was anticipated to depolarize CRF 
neurons and induces their firing. However, there have been no electrophysiological reports of NA activating directly an inward current or inducing depolarization in parvocellular neuroendocrine neurons [2]. Tasker and colleagues have proposed that NA may initiate signal transduction pathways without altering membrane conductance. Alternatively, postsynaptic NAergic receptors may stimulate synthesis of a retrograde messenger that could trigger the activation of upstream local glutamatergic circuits [2].

Although parvocellular neuroendocrine neurons have been characterized electrophysiologically, PVH-CRF neurons could not have been specified in previous studies. Very recently, it became possible to visualize CRF neurons labeled by fluorescent proteins in genetically engineered mouse lines (vide infra).

\section{Application of novel technologies for exploring CRF neurons}

\subsection{Labeling CRF neurons with fluorescent proteins}

In earlier studies, DNA sequences which determine the tissue-specific expression of the CRF gene were explored using transgenic mice lines, and very large regions of 5'- and/or 3'-flanking DNA sequences appear to be important for cell-specific expression and developmental regulation of CRF gene [57]. Cell-specific expression improved significantly with the inclusion of $8.7 \mathrm{~kb}$ of CRF $5^{\prime}$-flanking DNA, the entire CRF structural gene, and $2 \mathrm{~kb}$ of CRF $3^{\prime}$-flanking DNA [57], but even with the inclusion of $21 \mathrm{~kb}$ of $5^{\prime}$-flanking CRF DNA, the CRF transgene was not properly expressed compared to the endogenous CRF gene (Dr. Audrey Seasholtz, personal communication).

The BAC transgenic strategy was employed later in multiple laboratories. Alon and colleagues [19] reported a mouse line in which GFP was driven by CRF promoter (vide supra). GFP was expressed in brain regions of the transgenic mouse, and the anatomical distribution of the GFP-expressed neurons appeared mostly consistent with that reported in earlier studies using the rat [6-8]. These regions include the BNST, median/medial preoptic area, PVH, lateral hypothalamus, cingulate cortex, sensorimotor cortices, piriform cortex, CeA, LDTg, PPTg, PAG, Barrington's nucleus, LPB, NTS, and ventrolateral reticular formation. In addition to these regions, IO expressed prominent GFP-ir neurons, and CRF mRNA was also expressed in these regions $[19,20]$. The reason is not clear why IO was not described as one of the areas that express CRF-immunoreactivity conspicuously in the rat [6-8], and further examinations are required to examine whether this is due to the species difference. In the transgenic mouse line reported by Alon and colleagues [19], CRF expression was verified to be colocalized with GFP expression in the PVH. However, GFP expression was not intense enough to visualize $\mathrm{CRF}$ neurons under fluorescent microscope without immunostaining (personal communication), so this mouse line cannot be used for electrophysiological experiments.

Recently, Wamsteeker Cusulin and colleagues reported a mouse in which tdTomato is driven by the CAG promoter [18]. They crossed the CRH-IRES-Cre knock-in mouse [58] with a tdTomato reporter mouse, Ai14 [59], to be able to generate a mouse in which tdTomato fluorescent protein is expressed in the CRF neurons selectively. Itoi and colleagues also generated a novel knock-in mouse line, CRF-Venus, and succeeded in visualizing CRF neurons with Venus fluorescent protein [5].

\subsection{Generation of CRF-Venus knock-in mouse and CRF-iCre knock-in mouse}

Details of the methods for the generation of CRF-Venus knock-in mouse are described elsewhere [5]. The DNA constructs of the targeting vector is shown in Fig. 4. A DNA fragment, which carried Venus sequence and pgk-1 promoter-driven neomycin phosphotransferase gene (pgk-Neo) flanked by two flippase recognition target (frt) sites,

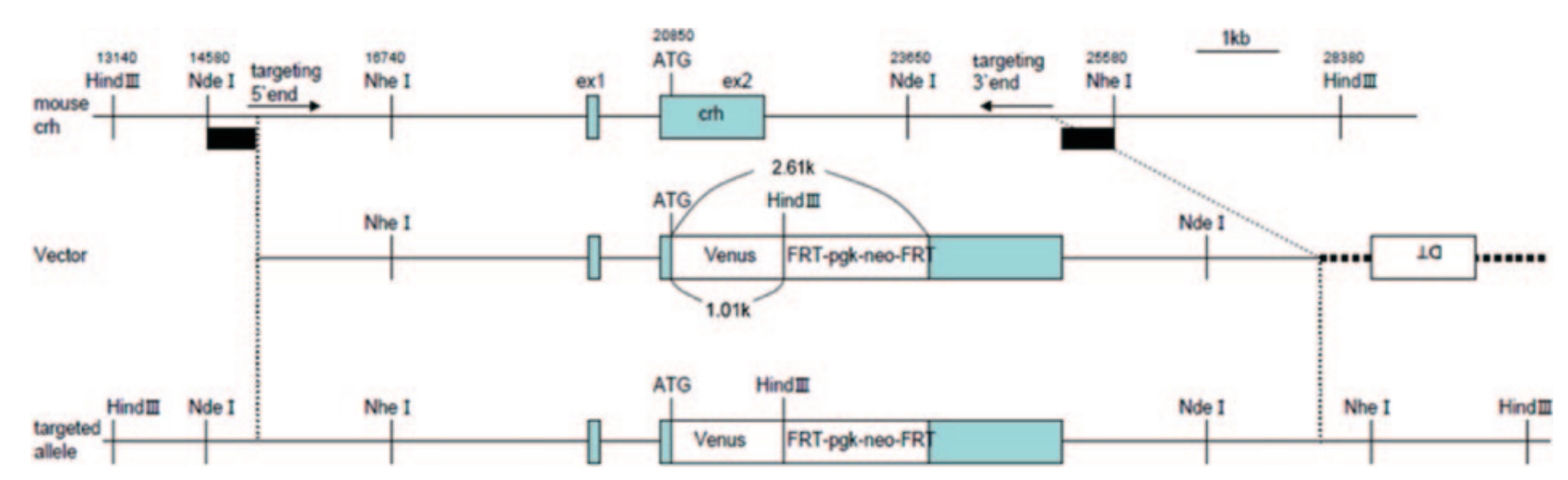

Fig. 4. DNA construct of targeting vector for generation of the CRF-Venus knock-in mouse

The mouse CRF genome, Venus vector, and Venus-targeted allele are shown. By homologous recombination, the Venus, together with the pgk-neomycin resistant cassette (pgk-neo), was inserted into exon 2 of the CRF gene in frame. The exons of the CRF gene are represented by colored boxes, while the Venus sequence, FRT-flanked pgk-neo, and diphtheria toxin (DT) cassette by open boxes. Closed boxes indicate 5'- and 3'-probe regions used for Southern blot analysis. Cleavage sites of restriction enzymes are also shown in the figure. 
was inserted into the translation initiation site of exon 2. The targeting vector was electroporated into the embryonic stem cell line RENKA [60], which is of C57BL/6N origin, and recombinant clones were identified by Southern blot analyses. It is noteworthy to mention that the backcross was unnecessary following the germ line transmission because the ES line of C57BL/6N origin (RENKA), and not of 129, was used in this study. Only the heterozygous mice were used in subsequent experiments. Itoi and colleagues [5] also generated the CRF-iCre knock-in mouse (CRF-iCre) with an identical strategy. The CRF-iCre is a driver mouse, and CRF neuron-selective knockout can be realized if a floxed line is available. CRF neuron-selective induction of a protein of interest may also be attained by crossing the driver with a reporter mouse line, in which the target sequence is flanked by a floxed-stop sequence, or local injection of a viral vector encoding an insert flanked by a floxed-stop sequence.

\subsection{Characterization of the CRF-Venus mouse and CRF-iCre mouse}

The Venus fluorescence was observed within the cytoplasm of neurons in the PVH of acute brain slices under a fluorescent microscope (Fig. 5A). The Venus-labeled neurons were also visible in other brain regions which are reported to express CRF-ir neurons in the rat [6-8] and CRF mRNA in mice [19]; these regions include the BNST, CeA, Barrington's nucleus and IO (not shown). We first examined whether the distribution of Venus-expressing cells was identical to that of CRF-expressing neurons.

A

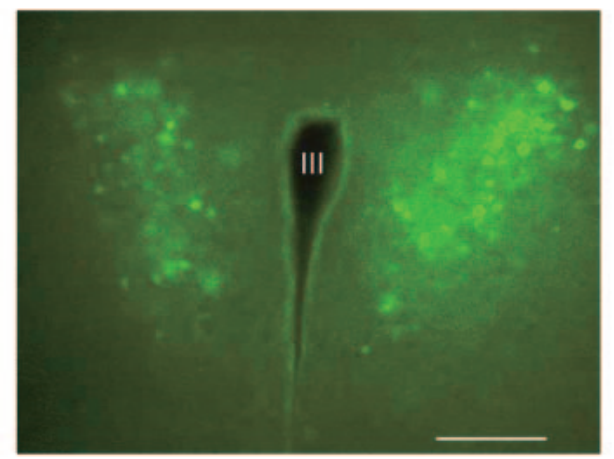

C

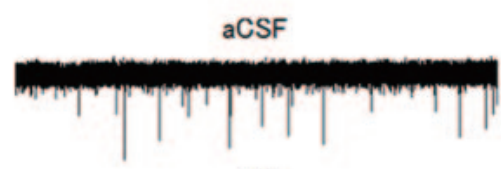

NA
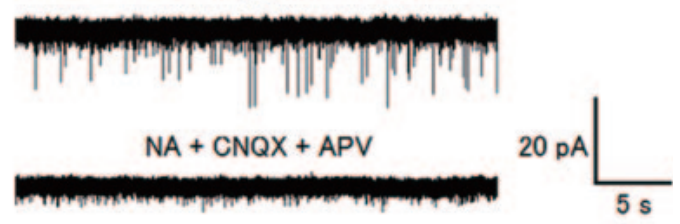

B

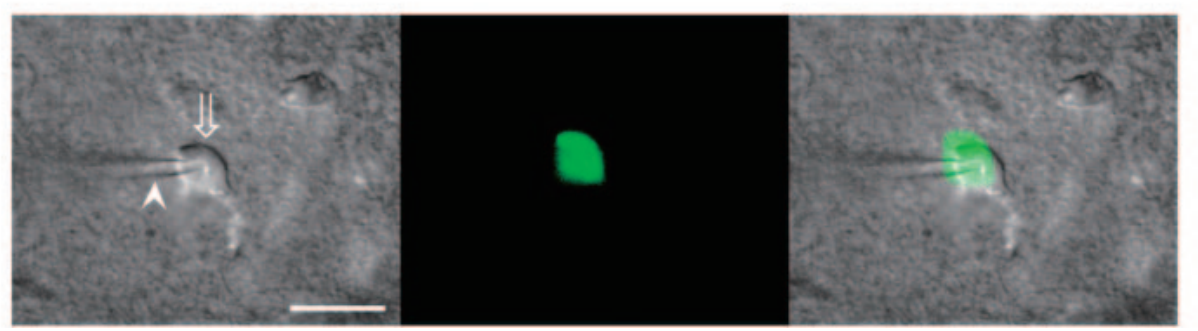

Fig. 5. Visualization of CRF neurons with Venus fluorescence and electrical recordings from the Venus-expressing neurons A. Venus-expressing neurons are observed under a fluorescent microscope in an acute hypothalamic slice without immunostaining. III, third ventricle. Scale bar $=200 \mu \mathrm{m}$. B. A glass pipette was made access to a Venus-expressing neuron, and conventional whole-cell clamp was performed. The arrowhead indicates the tip of the glass pipette, and the arrow the clamped cell. Scale bar $=20 \mu \mathrm{m}$. C. Upper panel: the slice was perfused with artificial cerebrospinal fluid (sCSF). Picrotoxin $(50 \mu \mathrm{M})$, a $\mathrm{GABA}_{\mathrm{A}}$ channel blocker, was applied to the bath solution. Under a voltage-clamp mode at $-60 \mathrm{mV}$, spontaneous EPSCs were recorded. Middle panel: noradrenalin (NA, $100 \mu \mathrm{M})$ increased the frequency of spontaneous EPSCs in approximately $60 \%$ of Venus-expressing neurons [5]. The amplitude and decay time of EPSCs were not affected significantly by NA (not shown). The lower panel: the bath application of $N$-methyl-D-aspartate (NMDA) receptor antagonist, D,L-2-amino-5phosphonovalerate (APV), and non-NMDA receptor antagonist, 6-cyano-7-nitroquinoxaline-2,3-dione (CNQX), abolished the EPSCs with or without (not shown) bath application of NA.

To make a precise anatomical localization of the Venus-expressing neurons, immunofluorescence histochemistry was carried out. Venus-expressing neurons were observed in the brain regions which were reported to contain CRF-ir neurons in the rat [6-8] and CRF mRNA in the mouse [19,20]. These regions include the BNST (dorsal and ventral), medial preoptic area, cerebral cortex (cingulate cortex, rostrocaudal extent of the sensorimotor cortices, mostly in layers II and III), piriform cortex, PVH, CeA, interstitial nucleus of the posterior limb of the anterior commissure 

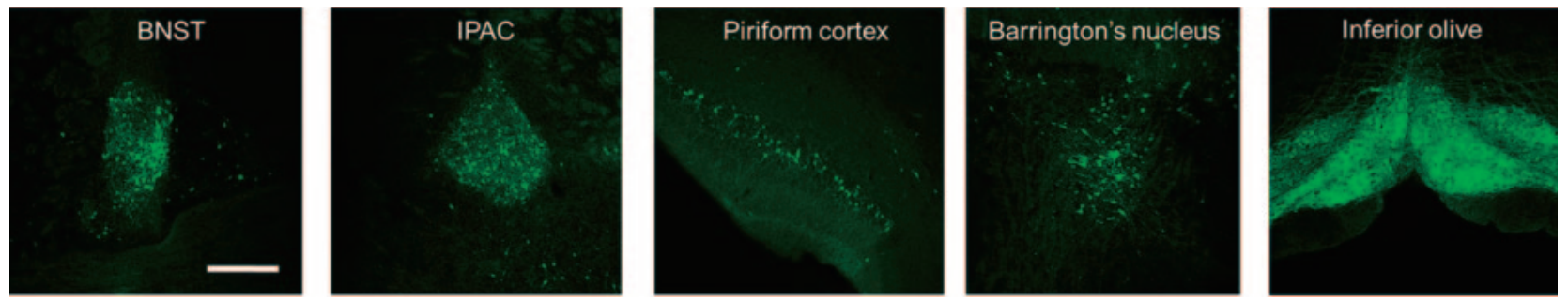

Fig. 6. Distribution of Venus-expressing neurons in brain regions outside the PVH of the CRF-Venus mouse

Distribution of Venus-expressing neurons in the brain regions outside the PVH were examined in the CRF-Venus knock-in mouse by immunofluorescence histochemistry. The Venus-expressing cells were observed in brain regions including the bed nucleus of the stria terminalis (BNST), CeA (not shown), interstitial nucleus of posterior limb of anterior commissure (IPAC), piriform cortex, Barrington's nucleus, and inferior olive. Bar $=400 \mu \mathrm{m}$.

(IPAC) (this nucleus has not been specified in the rat), LDTg, LPB, Barrington's nucleus, IO, and NTS (Fig. 6). As a whole, the regions which contained Venus-expressing neurons in the CRF-Venus mouse were similar to those which contained GFP-expressing neurons in the CRF-GFP transgenic mouse reported by Alon and colleagues [19].

Next, distribution of CRF-expressing neurons was compared to that of Venus-expressing neurons in the PVH by double immunofluorescence histochemistry (Fig. 7). Unexpectedly, there was a considerable inconsistency in distribution between Venus-expressing neurons and CRF-expressing neurons in a physiological state of a CRF-Venus mouse [5] (not shown). Partial inhibitory effect of circulating GC, on the Venus- or CRF gene expression, may underlie these phenomena. So, the co-localization of Venus with CRF was examined under a GC-deficient (adrenalectomized) condition; most Venus-expressing neurons co-expressed CRF under GC deficiency (Fig. 7). Thus, a partial suppression of the CRF promoter by physiological GC may have been related to the discrepancy between Venus expression and CRF expression in the CRF-Venus mouse.
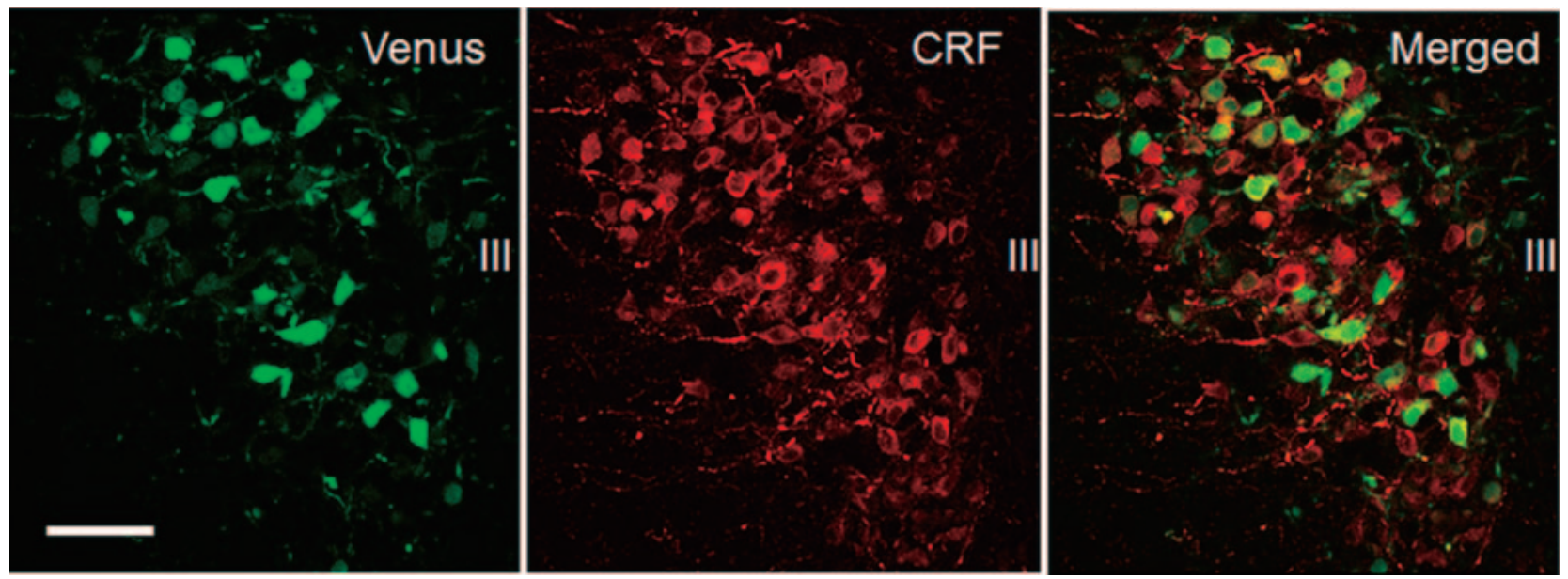

Fig. 7. Co-localization of Venus and CRF in the PVH of CRF-Venus mouse in a glucocorticoid-deficient state

Co-localization of Venus-expressing neurons and CRF-expressing neurons was examined by double immunofluorescence histochemistry in the PVH of CRF-Venus mouse after bilateral adrenalectomy. Most Venus-expressing neurons expressed CRF. Colchicine was injected intracerebroventricularly $24 \mathrm{~h}$ prior to sacrifice. III, the side facing the third ventricle, scale bar $=100 \mu \mathrm{m}$.

\subsection{Alternative methods to generate mice for visualization of CRF neurons}

In the mouse generated by Wamsteeker Cusulin and colleagues (crh-IRES-Cre;Ai14) [18], the tdTomato fluorescent protein is driven by the constitutive promoter, CAG, which is present in the upstream region of the tdTomato reporter mouse, Ai14 [59]. Double immunofluorescence histochemistry revealed that most CRF neurons in the PVH expressed tdTomato, and vice versa, indicating that tdTomato was expressed selectively in the PVH-CRF neurons [18]. The tdTomato expression was bright enough to be able to carry out electrophysiological studies.

With an identical strategy to that of Wamsteeker Cusulin and colleagues [18], Itoi and colleagues also generated another mouse, $\mathrm{Tg}^{\mathrm{CAG}-\mathrm{CAT}-\mathrm{EGFP} / \mathrm{wt}}$; $\mathrm{CRF}^{\mathrm{iCre} / \mathrm{wt}}$ (EGFP/CRF-iCre), in which enhanced green fluorescent protein (EGFP) was driven by the CAG promoter, by crossing the CRF-iCre knock-in mouse with the CAG-CAT-EGFP reporter [5]. In the PVH of the EGFP/CRF-iCre, EGFP was expressed more constitutively than Venus in the CRF-Venus mouse [5]. 
The reason is probably because their genes are driven by the constitutive bacterial promoter, CAG, and the transcriptional state is independent of the regulatory factors such as GC.

The genomic construct of CRF-Venus mouse was designed so that Venus expression was driven by the CRF promoter. This may be advantageous for monitoring dynamic changes in CRF neurons and CRF networks in different conditions, including pathological hyperglucocorticoid states (Cushing syndrome), hypoglucocorticoid states (Addison's disease), or acute or chronic stressful conditions. On the other hand, EGFP/CRF-iCre and crh-IRESCre;Ai14 have the advantage of being able to label CRF neurons more constitutively.

Recently, another mouse line, CRF-Venus $\Delta$ Neo, was generated (Itoi and colleagues, unpublished; reported in an abstract form at the Annual Meeting of Endocrine Society, 2015); the pgk-Neo cassette was deleted from the CRFVenus genome in the CRF-Venus $\triangle \mathrm{Neo}$. In the PVH of the CRF-Venus $\Delta$ Neo mouse, the Venus expression became more intense, and most Venus-expressing neurons co-expressed CRF, and vice versa (ibid). Thus, multiple animal resources are now available, and it is desirable to choose an appropriate mouse line(s) depending on the aim and design of each individual study.

\subsection{Future perspectives using novel animal tools}

Since the Venus-expressing (putative CRF) neurons were visualized under a fluorescent microscope, a glass pipette was made access to the Venus neuron, and conventional whole-cell patch-clamp recordings were made (Fig. 5B). Shown in Fig. 5C is a representative recording of glutamatergic EPSCs under a voltage-clam mode. The frequency of the glutamatergic EPSCs was increased by NA, and therefore, at least part of the excitatory action of NA may be mediated through the glutamatergic interneurons within the PVH (Fig. 5C). The effect of NA was inhibited by an $\alpha_{1}$-adrenergic receptor antagonist, prazosin (our unpublished observation). These results are consistent with those in previous studies in which the parvocellular neurons were examined in the rat and mouse $\mathrm{PVH}[55,56]$. GABAergic IPSCs were also observed in the Venus-expressing neurons (not shown). Wamsteeker Cusulin and colleagues also reported that the tdTomato-expressing (putative CRF) neurons in the PVH of the crh-IRES-Cre;Ai14, receive direct glutamatergic and GABAergic inputs [18].

The advent of the mouse lines, in which CRF neurons are visualized by fluorescent proteins, will contribute greatly to the development of the cellular physiological studies of CRF neurons. Using these animal tools, neurotransmitters and modulators that regulate the CRF neurons will be identified, and their mechanisms of actions on CRF neurons will be unraveled. These animals may also be used for studies aiming at elucidating the mechanisms of excitability, signal transduction, and genomic expression in the CRF neurons.

Wamsteeker Cusulin and colleagues were able to express channelrhodopsin 2 (ChR2) by local microinjection of an adeno-associated virus containing a floxed ChR2 construct into the PVH of a crf-IRES-Cre mouse [18]. They could elicit firing of the putative CRF neurons by delivering 473-nm blue light pulses to the slice. CRF neuron-selective expression of channelrhodopsins or halo-/archeo-rhodopsins [61] will enable us to excite or silence CRF neurons in either in vitro or in vivo experimental setups. In addition, a gene of interest can be knocked out or induced in a CRF neuron-selective manner, as was described above. Taken together, these novel mouse tools will enable us to explore the mechanisms for the control of the HPA-axis from both the cellular and systemic standpoints and to elucidate how it functions to defend an organism from various stressors and maintain the homeostatic balance.

\section{Summary}

In the present review, I intended to help readers understand the characteristics of the mouse lines generated in my laboratory, and hopefully, encourage them to use these animals in future studies for unraveling the cellular mechanisms for CRF neurons in the PVH (and perhaps outside the PVH as well). Attempts to label CRF neurons have been made since two decades ago, but the tissue- (or cell-) specific targeting of the CRF gene was no easy task. The BAC transgenic strategy [62] became widely used later, and the tissue-selective expression of transgenes appears much improved. As far as the CRF gene is concerned, however, even with the insertion of the long promoter regions contained in the BAC clone(s), the transgene(s) were not expressed 'correctly' in tissues where CRF gene ought to be expressed. Recently, CRF neurons became labeled by fluorescent proteins by the knock-in strategy [5,18]. CRF-Venus and CRF-Venus $\Delta$ Neo mouse lines will be of great benefit in uncovering the unsolved problems related to CRF neurons. CRF-driver mouse lines, crh-IRES-Cre and CRF-iCre, were generated by a similar strategy. Besides their use for obtaining a mouse that express fluorescent markers $[5,18]$, they will also be utilized in conjunction with other technologies such as the gene transfer by viral vectors (e.g., transfer of channelrhodopsin genes for the purpose of stimulating or silencing CRF neurons in vitro and in vivo), activation or suppression of CRF neurons by designer receptors exclusively activated by designer drugs (DREADD) [63], or CRF-neuron selective knockouts.

\section{Acknowledgment}

The CRF-Venus knock-in mouse and CRF-iCre-knock-in mouse were generated with the collaboration of Dr. Kenji Sakimura, Brain Research Institute, Niigata University, Japan. The scholars and students who participated in generation 
and characterization of the knock-in mice were Toshimitsu Fuse, Takuji Kaneko, Ryo Ozawa, Takayuki Sato, Takuma Sugaya, Naoya Sugimoto, Katsuya Uchida, Junko Kono, Maya Yamazaki, Manabu Abe, and Rie Natsume. I thank Dr. Jun-ichi Miyazaki, Osaka University, Japan, for generously providing us with the CAG-CAT-EGFP reporter mice. Part of this work was supported by research grants from JSPS. Disclosure Summary: The author has nothing to disclose.

\section{REFERENCES}

[1] Itoi K, Jiang YQ, Iwasaki Y, Watson SJ (2004) Regulatory mechanisms of corticotropin-releasing hormone and vasopressin gene expression in the hypothalamus. J Neuroendocrinol 16:348-355.

[2] Levy BH, Tasker JG (2012) Synaptic regulation of the hypothalamic-pituitary-adrenal axis and its modulation by glucocorticoids and stress. Front Cell Neurosci:6:24.

[3] Ulrich-Lai YM, Herman JP (2009) Neural regulation of endocrine and autonomic stress responses. Nat Rev Neurosci 10:397-409.

[4] Son SJ, Filosa JA, Potapenko ES, Biancardi VC, Zheng H, Patel KP, Tobin VA, Ludwig M, Stern JE (2013) Dendritic peptide release mediates interpopulation crosstalk between neurosecretory and preautonomic networks. Neuron 78:1036-1049.

[5] Itoi K, Talukder AH, Fuse T, Kaneko T, Ozawa R, Sato T, Sugaya T, Uchida K, Yamazaki M, Abe M, Natsume R, Sakimura K (2014) Visualization of corticotropin-releasing factor neurons by fluorescent proteins in the mouse brain and characterization of labeled neurons in the paraventricular nucleus of the hypothalamus. Endocrinology 155:4054-4060.

[6] Swanson LW, Sawchenko PE, Rivier J, Vale WW (1983) Organization of ovine corticotropin-releasing factor immunoreactive cells and fibers in the rat brain: an immunohistochemical study. Neuroendocrinology 36:165-86.

[7] Merchenthaler I (1984) Corticotropin releasing factor (CRF)-like immunoreactivity in the rat central nervous system. Extrahypothalamic distribution. Peptides 5 Suppl 1:53-69.

[8] Sakanaka M, Shibasaki T, Lederis K (1987) Corticotropin releasing factor-like immunoreactivity in the rat brain as revealed by a modified cobalt-glucose oxidase-diaminobenzidine method. J Comp Neurol 260:256-298.

[9] Merchenthaler I, Vigh S, Petrusz P, Schally AV (1983) The paraventriculo-infundibular corticotropin releasing factor (CRF) pathway as revealed by immunocytochemistry in long-term hypophysectomized or adrenalectomized rats. Regul Pept 5:295-305.

[10] Vale W, Spiess J, Rivier C, Rivier J (1981) Characterization of a 41-residue ovine hypothalamic peptide that stimulates secretion of corticotropin and beta-endorphin. Science 213:1394-1397.

[11] Valentino RJ, Wood SK, Wein AJ, Zderic SA (2011) The bladder-brain connection: putative role of corticotropin-releasing factor. Nat Rev Urol 8:19-28.

[12] Miyata M, Okada D, Hashimoto K, Kano M, Ito M (1999) Corticotropin-releasing factor plays a permissive role in cerebellar long-term depression. Neuron 22:763-775.

[13] Mouri T, Suda T, Sasano N, Andoh N, Takei Y, Takase M, Sasaki A, Murakami O, Yoshinaga K (1984) Immunocytochemical identification of CRF in the human hypothalamus. Tohoku J Exp Med 142:423-426.

[14] Mouri T, Itoi K, Takahashi K, Suda T, Murakami O, Yoshinaga K, Andoh N, Ohtani H, Masuda T, Sasano N (1993) Colocalization of corticotropin-releasing factor and vasopressin in the paraventricular nucleus of the human hypothalamus. Neuroendocrinology 57:34-39.

[15] Pelletier G, Désy L, Côté J, Vaudry H (1983) Immunocytochemical localization of corticotropin-releasing factor-like immunoreactivity in the human hypothalamus. Neurosci Lett 41:259-263.

[16] Takahashi K, Mouri T, Yamamoto T, Itoi K, Murakami O, Yoshinaga K, Sasano N (1989) Corticotropin-releasing hormone in the human hypothalamus. Free-floating immunostaining method. Endocrinol Japon 36, 275-280.

[17] Mihály E, Fekete C, Lechan RM, Liposits Z (2002) Corticotropin-releasing hormone-synthesizing neurons of the human hypothalamus receive neuropeptide Y-immunoreactive innervation from neurons residing primarily outside the infundibular nucleus. J Comp Neurol 446:235-243.

[18] Wamsteeker Cusulin JI, Füzesi T, Watts AG, Bains JS (2013) Characterization of corticotropin-releasing hormone neurons in the paraventricular nucleus of the hypothalamus of Crh-IRES-Cre mutant mice. PLoS One 8:e64943.

[19] Alon T, Zhou L, Pérez CA, Garfield AS, Friedman JM, Heisler LK (2009) Transgenic mice expressing green fluorescent protein under the control of the corticotropin-releasing hormone promoter. Endocrinology 150:5626-5632.

[20] Keegan CE, Herman JP, Karolyi IJ, O'Shea KS, Camper SA, Seasholtz AF (1994) Differential expression of corticotropinreleasing hormone in developing mouse embryos and adult brain. Endocrinology 134:2547-2555.

[21] Simmons DM, Swanson LW (2009) Comparison of the spatial distribution of seven types of neuroendocrine neurons in the rat paraventricular nucleus: toward a global 3D model. J Comp Neurol 516:423-441.

[22] Armstrong WE, Warach S, Hatton GI, McNeill TH (1980) Subnuclei in the rat hypothalamic paraventricular nucleus: a cytoarchitectural, horseradish peroxidase and immunocytochemical analysis. Neuroscience 5:1931-1958.

[23] Swanson LW, Kuypers HG (1980) The paraventricular nucleus of the hypothalamus: cytoarchitectonic subdivisions and organization of projections to the pituitary, dorsal vagal complex, and spinal cord as demonstrated by retrograde fluorescence double-labeling methods. J Comp Neurol 194:555-570.

[24] Sawchenko PE, Swanson LW (1982) Immunohistochemical identification of neurons in the paraventricular nucleus of the hypothalamus that project to the medulla or to the spinal cord in the rat. J Comp Neurol 205:260-272.

[25] Dampney RA (1994) Functional organization of central pathways regulating the cardiovascular system. Physiol Rev 74:323-364.

[26] Biag J, Huang Y, Gou L, Hintiryan H, Askarinam A, Hahn JD, Toga AW, Dong HW (2012) Cyto- and chemoarchitecture of the hypothalamic paraventricular nucleus in the C57BL/6 J male mouse: a study of immunostaining and multiple fluorescent tract tracing. J Comp Neurol 520:6-33. 
[27] Tramu G, Croix C, Pillez A (1983) Ability of the CRF immunoreactive neurons of the paraventricular nucleus to produce a vasopressin-like material. Immunohistochemical demonstration in adrenalectomized guinea pigs and rats. Neuroendocrinology 37:467-469.

[28] Kiss JZ, Mezey E, Skirboll L (1984) Corticotropin-releasing factor-immunoreactive neurons of the paraventricular nucleus become vasopressin positive after adrenalectomy. Proc Natl Acad Sci U S A 81:1854-1858.

[29] Itoi K, Mouri T, Takahashi K, Murakami O, Imai Y, Sasaki S, Yoshinaga K, Sasano N (1987) Suppression by glucocorticoid of the immunoreactivity of corticotropin-releasing factor and vasopressin in the paraventricular nucleus of rat hypothalamus. Neurosci Lett 73:231-236.

[30] Whitnall MH, Mezey E, Gainer H (1985) Co-localization of corticotropin-releasing factor and vasopressin in median eminence neurosecretory vesicles. Nature 317:248-250.

[31] Murakami K, Hashimoto K, Ota Z (1984) Interaction of synthetic ovine corticotropin releasing factor and arginine vasopressin on in vitro ACTH release by the anterior pituitary of rats. Neuroendocrinology 39:49-53.

[32] Rivier C, Rivier J, Mormede P, Vale W (1984) Studies of the nature of the interaction between vasopressin and corticotropinreleasing factor on adrenocorticotropin release in the rat. Endocrinology 115:882-886.

[33] Daftary SS, Boudaba C, Szabó K, Tasker JG (1998) Noradrenergic excitation of magnocellular neurons in the rat hypothalamic paraventricular nucleus via intranuclear glutamatergic circuits. J Neurosci 18:10619-10628.

[34] Boudaba C, Tasker JG (2006) Intranuclear coupling of hypothalamic magnocellular nuclei by glutamate synaptic circuits. Am J Physiol 291:R102-R111.

[35] Di S, Malcher-Lopes R, Halmos KC, Tasker JG (2003) Nongenomic glucocorticoid inhibition via endocannabinoid release in the hypothalamus: a fast feedback mechanism. J Neurosci 23:4850-4857.

[36] Boudaba C, Szabó K, Tasker JG (1996) Physiological mapping of local inhibitory inputs to the hypothalamic paraventricular nucleus. J Neurosci 16:7151-7160.

[37] Pacák K, Palkovits M (2001) Stressor specificity of central neuroendocrine responses: implications for stress-related disorders. Endocr Rev 22:502-548.

[38] Itoi K, Sugimoto N (2010) The brainstem noradrenergic systems in stress, anxiety and depression. J Neuroendocrinol 22:355-361.

[39] Cunningham ET Jr, Sawchenko PE (1988) Anatomical specificity of noradrenergic inputs to the paraventricular and supraoptic nuclei of the rat hypothalamus. J Comp Neurol 274:60-76.

[40] Uchida K, Kobayashi D, Das G, Onaka T, Inoue K, Itoi K (2010) Participation of the prolactin-releasing peptide-containing neurones in caudal medulla in conveying haemorrhagic stress-induced signals to the paraventricular nucleus of the hypothalamus. J Neuroendocrinol 22:33-42.

[41] Radley JJ, Williams B, Sawchenko PE (2008) Noradrenergic innervation of the dorsal medial prefrontal cortex modulates hypothalamo-pituitary-adrenal responses to acute emotional stress. J Neurosci 28:5806-5816.

[42] Malcher-Lopes R, Franco A, Tasker JG (2012) Glucocorticoids shift arachidonic acid metabolism toward endocannabinoid synthesis: a non-genomic anti-inflammatory switch. Eur J Pharmacol 583:322-339.

[43] Hill MN, Tasker JG (2012) Endocannabinoid signaling, glucocorticoid-mediated negative feedback, and regulation of the hypothalamic-pituitary-adrenal axis. Neuroscience 204:5-16.

[44] Aguilera G (2015) Molecular Regulation of Corticotropin-Releasing Hormone Gene Expression in Parvocellular Neurons of the Hypothalamic Paraventricular Nucleus. Interdisciplinary Information Sciences 21:273-282.

[45] Tasker JG, Dudek FE (1991) Electrophysiological properties of neurones in the region of the paraventricular nucleus in slices of rat hypothalamus. J Physiol 434:271-93.

[46] Luther JA, Tasker JG (2000) Voltage-gated currents distinguish parvocellular from magnocellular neurones in the rat hypothalamic paraventricular nucleus. J Physiol 523:193-209.

[47] Luther JA, Daftary SS, Boudaba C, Gould GC, Halmos KC, Tasker JG (2002) Neurosecretory and non-neurosecretory parvocellular neurones of the hypothalamic paraventricular nucleus express distinct electrophysiological properties. J Neuroendocrinol 14:929-932.

[48] Stern JE (2001) Electrophysiological and morphological properties of pre-autonomic neurones in the rat hypothalamic paraventricular nucleus. J Physiol 537:161-177.

[49] Yang JH, Li LH, Shin SY, Lee S, Lee SY, Han SK, Ryu PD (2008) Adrenalectomy potentiates noradrenergic suppression of GABAergic transmission in parvocellular neurosecretory neurons of hypothalamic paraventricular nucleus. J Neurophysiol 99:514-523.

[50] Füzesi T, Wittmann G, Liposits Z, Lechan RM, Fekete C (2007) Contribution of noradrenergic and adrenergic cell groups of the brainstem and agouti-related protein-synthesizing neurons of the arcuate nucleus to neuropeptide-y innervation of corticotropin-releasing hormone neurons in hypothalamic paraventricular nucleus of the rat. Endocrinology 148:5442-50.

[51] Liposits Z, Sherman D, Phelix C, Paull WK (1986) A combined light and electron microscopic immunocytochemical method for the simultaneous localization of multiple tissue antigens. Tyrosine hydroxylase immunoreactive innervation of corticotropin releasing factor synthesizing neurons in the paraventricular nucleus of the rat. Histochemistry 85:95-106.

[52] Ericsson A, Kovács KJ, Sawchenko PE (1994) A functional anatomical analysis of central pathways subserving the effects of interleukin-1 on stress-related neuroendocrine neurons. J Neurosci 14:897-913.

[53] Itoi K, Suda T, Tozawa F, Dobashi I, Ohmori N, Sakai Y, Abe K, Demura H (1994) Microinjection of norepinephrine into the paraventricular nucleus of the hypothalamus stimulates corticotropin-releasing factor gene expression in conscious rats. Endocrinology 135:2177-2182.

[54] Itoi K, Helmreich DL, Lopez-Figueroa MO, Watson SJ (1999) Differential regulation of corticotropin-releasing hormone and vasopressin gene transcription in the hypothalamus by norepinephrine. J Neurosci 19:5464-5472.

[55] Yang JH, Li LH, Lee S, Jo IH, Lee SY, Ryu PD (2007) Effects of adrenalectomy on the excitability of neurosecretory 
parvocellular neurones in the hypothalamic paraventricular nucleus. J Neuroendocrinol 19:293-301.

[56] Daftary SS, Boudaba C, Tasker JG (2000) Noradrenergic regulation of parvocellular neurons in the rat hypothalamic paraventricular nucleus. Neuroscience 96:743-751.

[57] Keegan CE, Karolyi IJ, Knapp LT, Bourbonais FJ, Camper SA, Seasholtz AF (1994) Expression of corticotropin-releasing hormone transgenes in neurons of adult and developing mice. Mol Cell Neurosci 5:505-514.

[58] Taniguchi H, He M, Wu P, Kim S, Paik R, Sugino K, Kvitsiani D, Fu Y, Lu J, Lin Y, Miyoshi G, Shima Y, Fishell G, Nelson SB, Huang ZJ (2011) A resource of Cre driver lines for genetic targeting of GABAergic neurons in cerebral cortex. Neuron 71:995-1013.

[59] Madisen L, Zwingman TA, Sunkin SM, Oh SW, Zariwala HA, Gu H, Ng LL, Palmiter RD, Hawrylycz MJ, Jones AR, Lein ES, Zeng H (2010) A robust and high-throughput Cre reporting and characterization system for the whole mouse brain. Nat Neurosci 13:133-140.

[60] Mishina M, Sakimura K (2007) Conditional gene targeting on the pure C57BL/6 genetic background. Neurosci Res 58:105-112.

[61] Madisen L, Mao T, Koch H, Zhuo JM, Berenyi A, Fujisawa S, Hsu YW, Garcia AJ 3rd, Gu X, Zanella S, Kidney J, Gu H, Mao Y, Hooks BM, Boyden ES, Buzsáki G, Ramirez JM, Jones AR, Svoboda K, Han X, Turner EE, Zeng H (2012) A toolbox of Cre-dependent optogenetic transgenic mice for light-induced activation and silencing. Nat Neurosci 15:793-802.

[62] Yang XW, Model P, Heintz N (1997) Homologous recombination based modification in Escherichia coli and germline transmission in transgenic mice of a bacterial artificial chromosome. Nat Biotechnol 15:859-865.

[63] Krashes MJ, Koda S, Ye C, Rogan SC, Adams AC, Cusher DS, Maratos-Flier E, Roth BL, Lowell BB (2011) Rapid, reversible activation of AgRP neurons drives feeding behavior in mice. J Clin Invest 121:1424-1428. 